\title{
JET-X \\ A JOINT EUROPEAN X-RAY TELESCOPE FOR SPECTRUM-X
}

\author{
A. Wells ${ }^{1}$, D.H. Lumb ${ }^{1}$, K.A. Pounds ${ }^{1}$,G.C. Stewart ${ }^{1}$, B. Aschenbach ${ }^{2}$, \\ H. Brauninger ${ }^{2}$, G. Hasinger ${ }^{2}$, J. Trumper ${ }^{2}$, O. Citterio ${ }^{3}$, L. Scarsi ${ }^{4}$, \\ A. Peacock ${ }^{5} \&$ B. Taylor 5 .
}

\author{
${ }^{1}$ Physics Department, Leicester University, Leicester, U.K. \\ ${ }^{2}$ Max Planck Institute fur Extraterrestriche Physik, Garching, W. Germany. \\ ${ }^{3}$ Osservatorio Astronomica di Brera, Merate, Italy. \\ ${ }^{4}$ Instituto Fisica Cosmica e Informatica del CNR, Palermo, Italy. \\ ${ }^{5}$ Space Science Department, ESTEC, Netherlands.
}

\begin{abstract}
The Joint European X-Ray Telescope, JET-X, is one of the core instruments of the scientific payload of the USSR SPECTRUM-X astrophysics mission due for launch in 1993. The JET-X instrument concept is described and its scientific performance and capability discussed.
\end{abstract}

\section{INTRODUCTION}

$\mathrm{X}$-ray emission constitutes an important fraction, often most, of the energy emitted from whole classes of astronomical objects ranging from stars to distant clusters of galaxies and the cosmic $\mathrm{X}$-ray background. The Joint European X-ray Telescope, JET-X, is designed to study the X-ray emission from these sources in the band $0.15-10 \mathrm{keV}$; particularly to meet primary scientific goals in cosmology and extragalactic astronomy. JET-X has been selected for the core payload of the USSR's SPECTRUM-X Project whose combined response extends over the range $20 \mathrm{eV}-100 \mathrm{keV}$.

JET-X is being developed by a consortium from the UK groups from the Universities of Leicester and Birmingham; the Rutherford Appleton Laboratory; the Mullard Space Science Laboratory; the Max Planck Institut for Extraterrestrial Physics, Garching, W. Germany; groups from the CNR and Universities in Milan, Rome and Palermo, Italy and the Space Science Department of ESTEC, ESA.

JET-X consists of two identical, coaligned X-ray imaging telescopes, each with a spatial resolution of $\mathbf{3 0}$ arcsecond or better. Focal plane imaging is provided by a cooled X-ray sensitive CCD detector which will combine high spatial resolution with good spectral resolution, with particular emphasis on high sensitivity and spectral resolution $\left(\frac{E}{\Delta E} \geq 50\right)$ around the $7 \mathrm{keV}$ Fe-line complex. An optical monitor is co-mounted with the $\mathrm{X}$-ray telescopes to permit simultaneous observation and identification of the optical counterparts of X-ray target sources. 
JET-X is conceived as a stand-alone instrument with simple interfaces to the SPECTRUM-X spacecraft. Development risks have been minimised by selecting mature technology for the mirror and detector systems; the mirrors will be produced using the replication techniques already under development for the Italian SAX mission, and the detectors will be based on development from current Leicester and ESTEC CCD programmes. JET-X will generate its own aspect solution, through pointing measurements with an integrally mounted attitude monitor and incorporates onboard data handling electronics for controlling the instrument with mass memory sufficient to store 24 hours-worth of scientific data. Data will be read out, on command, by the spacecraft on-board computer and transmitted to the ground receiving station via the spacecraft telemetry system during ground contacts.

\section{INSTRUMENT DESCRIPTION}

\subsection{Design Objectives}

The primary objectives of JET-X are:

1. Imaging with $\leq \mathbf{3 0}$ arcsec resolution with a limiting sensitivity at $1 \mathrm{keV}$ of $\sim 0.5 \mathrm{nJy}$

2. Medium resolution spectroscopy $\left(\frac{E}{\Delta E} \geq 10\right)$ in the $1-10 \mathrm{keV}$ band with emphasis on high sensitivity and spectral resolution $\left(\frac{E}{\Delta E} \geq 50\right)$ around the $7 \mathrm{keV}$ Fe-line complex.

3. Time variability of $\mathrm{X}$-ray spectra on timescales ranging from milliseconds to months.

4. Simultaneous (and continuous) optical monitoring of the $\mathrm{X}$-ray sources to a limiting magnitude of $\mathrm{m}_{\mathrm{v}} \sim 22$.

The limiting $\mathrm{x}$-ray sensitivity will be $\sim 3$ orders of magnitude better than previous sky-survey instruments, better than that of the EINSTEIN Observatory (by 1 order of magnitude) and comparable to that of the ROSAT XRT but with a wider energy range. Observations with JET-X will be of significant importance for cosmological studies. The instrument will be able to study the emission from large, statistically significant, samples of all the known classes of X-ray source. JET-X provides one of the first opportunities in X-ray astronomy to use a CCD detector at the focus of a high resolution imaging telescope. The energy resolution is sufficiently high to use the diagnostic emission and absorption features in the source spectra to study the emission mechanisms, chemical composition and the environment of cosmic sources in more detail than has hitherto been possible.

Simultaneous and complementary observations can be carried out using any of the other instruments in the SPECTRUM-X payload. For example, low energy (0.1-1.0 keV) imaging photometry with moderate spectral resolution using JET-X can be combined with the imaging continuum spectrophotometry offered by proposed extreme ultraviolet imaging instruments. Alternatively, simultaneous spectrophotometry and imaging with JET-X can be combined with time-resolved spectroscopic studies with the large area, lower resolution foil telescope X-SPECT instrument provided by IKI and the Danish Space Research Institute. Naturally, response overlaps between the different instruments allow for simultaneous cross-calibration of the responses in the overlapping wavebands. 


\subsection{Instrument Concept}

Two identical telescopes, each with a nested array of 12 mirrors, aperture of $0.3 \mathrm{~m}$ and a focal length of $3.5 \mathrm{~m}$, have a total effective area of $360 \mathrm{~cm}^{2}$ at $1.5 \mathrm{keV}, 140 \mathrm{~cm}^{2}$ at $8 \mathrm{keV}$. The angular resolution will be better than 30 arcseconds with a design potential of 10 arcseconds. Figure 1 shows the effective collecting area over the energy range.

The focal plane detector provides a spatial resolution compatible with the optics ( $<100$ microns), together with broad band spectroscopy with a resolution in the $0.15-10 \mathrm{keV}$ energy band in the range 5 to 60. A CCD instrument has been chosen for its potential to provide the necessary energy resolution, simultaneously with good quantum detection efficiency and background rejection, together with a spatial resolution which suitably oversamples the telescope angular response function.

Front-illuminated (FI) three-phase CCDs covering the energy band $0.8-10 \mathrm{keV}$ have already been developed to a sufficiently advanced stage to meet the requirements for the JET-X instrument (Chowanietz, Lumb and Wells, 1986). These devices are fabricated on high resistivity (1000 ohm$\mathrm{cm}$ ) silicon to obtain good quantum detection efficiency at energies above $3 \mathrm{keV}$. A back-illuminated (BI) version is now under development using higher resistivity epitaxial silicon, to further enhance the higher energy response, and allow the energy response to be extended down to $0.2 \mathrm{keV}$.

A $3 \times 3$ array of CCDs covers a field of view of $40 \operatorname{arcmin} \times 30$ arcmin in the field of view of each telescope but dead spaces between the individual CCDs limit the field coverage to $75 \%$. By mounting the arrays at right angles to each other in the two telescopes, an overall field coverage of $95 \%$ is achieved with $20 \%$ of the field covered at reduced efficiency. With the CCDs cooled to around $170-180 \mathrm{~K}$, energy resolution of $150 \mathrm{eV} \mathrm{FWHM}$ at $8 \mathrm{keV}$ can be achieved over the whole field of view. The quantum detection efficiency and other device parameters are summarised in Section 2.4 .

An option under consideration is to mount a retractable grazing incidence synthetic multi-layer structure in one telescope to provide high dispersion in the vicinity of the iron $\mathrm{K}$ - line $\left(\frac{E}{\Delta E} \geq 350\right)$, with the CCD acting as the readout for the dispersed spectrum.

The two telescopes will be co-aligned in a common structure, together with an attitude monitor which will provide an independent attitude solution to 5 arcsec accuracy, or alternatively to provide spacecraft attitude correction data for JET-X pointings. An optical monitor with sensitivity down to $\mathrm{m}_{\mathrm{v}}=22$ to allow simultaneous optical observation on the chosen X-ray targets is currently under study. Electronics units drive the CCD detectors, digitize and process the $\mathrm{x}$-ray images and deliver digital science data to a JET-X central data management unit which monitors the JET-X instrument status and housekeeping and controls the optical and attitude monitors.

The SPECTRUM-X data handling system consists of a spacecraft on-board computer (OBC) which can combine data from up to 16 separate experiments. The $O B C$ will control data handling for downlink and provide instrument control through up-linked commands. Telemetry is provided via a low speed link (up to $65 \mathrm{~kb} / \mathrm{s}$ ) for command verification and housekeeping and a high speed link (up to $18 \mathrm{Mb} / \mathrm{s}$ ) for science data. Telemetry and telecommand links are for up to 1.5 hours daily during normal mission operations. These operational constraints mean the instrument requires its own on-board mass memory with capacity to store 24-hours worth of science data. The JET-X mass memory will be part of the central data handling unit and will be read-out on command by the spacecraft $\mathrm{OBC}$ during ground contacts. 


\subsection{Telescope Mirrors and Mirror Mount}

Arrays of nested electroformed mirror shells, using Wolter-I geometry, are used to form the x-ray imaging optics for JET-X. The construction technique is based on replication in which a mandrel is machined to the required paraboloid-hyperboloid figure and then super-polished to a surface finish of around $5 \AA \mathrm{rms}$. A gold layer is deposited (by sputtering or evaporation) on to the mandrel surface, and a nickel layer is electroformed on top of this. Next the nickel shell is separated from the mandrel and the gold layer adheres to the nickel.

This technique has already reached an advanced state of development for the Italian SAX programme, where nickel replica mirrors $1 \mathrm{~mm}$ thick and with diameters of $165 \mathrm{~mm}$ and $65 \mathrm{~mm}$ have been manufactured and shown to have an angular resolution (HPW) of 60 arcsec at $8 \mathrm{keV}$ (limited mainly by the conical approximation) when mounted in a nested pair (Citterio et al., 1987). For JET-X, two modifications to the SAX technique are being pursued which will lead to the attainment of better intrinsic angular resolution whilst enabling larger diameter mirrors to be produced. The conical approximation of SAX will be replaced by a true Wolter I configuration. This will ensure that on-axis resolution is dominated by figure errors and surface finish only. The target specification per mirror is 5 arcseconds. Secondly, the nickel layers will be made thicker $(2 \mathrm{~mm})$ so as to ensure better stability, especially with the larger mirrors.

The mirror parameters are summarised in Table 1. Figure 2 shows the mirror mounting system.

\section{Table 1 Mirror Characteristics}

$\begin{array}{ll}\text { Field of View } & 20 \operatorname{arcmin}(50 \% \text { vignetting) } \\ \text { Angular resolution } & 10-30 \operatorname{arcsec}(\mathrm{HPW}) \\ \text { Focal length } & 3.5 \mathrm{~m} \\ \text { Reflecting surface } & \text { Gold } \\ \text { Configuration } & \text { Wolter-I } \\ \text { Mirror length } & 2 \times 300 \mathrm{~mm} \\ \text { Outer mirror diameter } & 300 \mathrm{~mm} \\ \text { Inner mirror diameter } & 87.5 \mathrm{~mm} \\ \text { Shell thickness } & 2 \mathrm{~mm} \\ \text { Number of shells } & 12 \\ \text { Distance between shells } & \mathbf{3 - 5 . 8 \mathrm { mm }} \\ \text { Surface finish (microroughness) } & 5 \AA \mathrm{rms}\end{array}$

\subsection{Focal Instrument Assembly}

Development of a $3 \times 3$ array of fully depleted back-illuminated CCDs is currently in progress. Performance data has so far been obtained on front-illuminated fully depleted devices (Lumb and Holland, 1989). The imaging capability was demonstrated by resolution tests using a mask with an array of 150 micron pinholes spaced $0.5 \mathrm{~mm}$ apartand and illuminated with $1.5 \mathrm{keV}$ radiation. Resolution down to an equivalent telescope HPW of 10 " was achieved.

Figure 3 compares the predicted efficiency for a 30 micron depletion depth with measured data with total detection efficiency (including split events) and detection efficiency for isolated pixels, both being recorded. The good spectroscopic response of the CCD is demonstrated by the similarity 


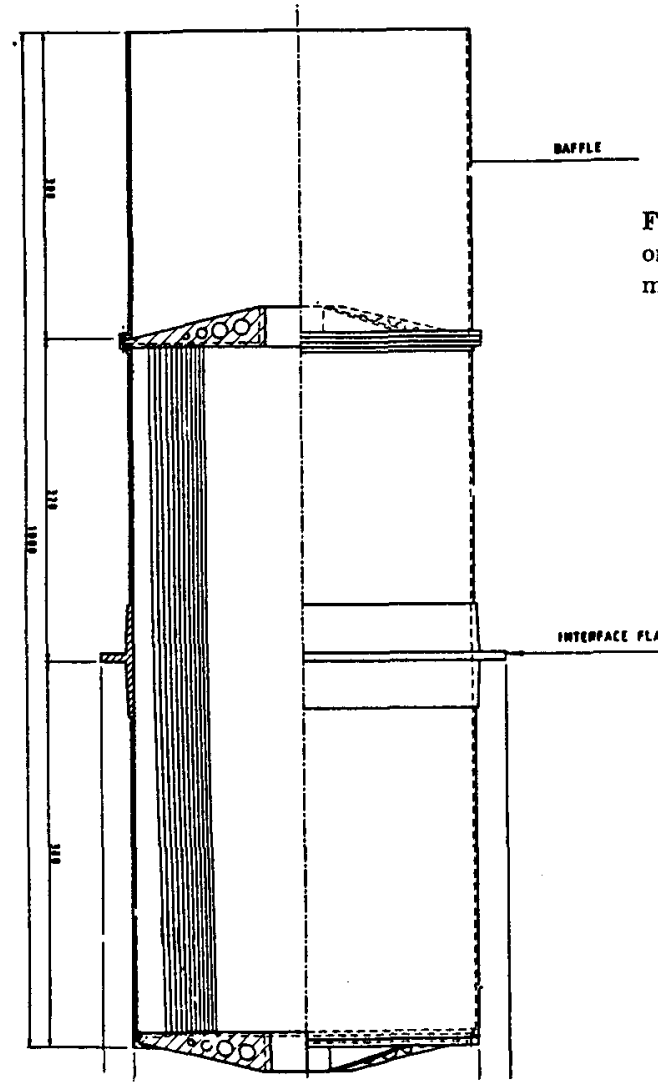

Figure 2: Design for the mirror mounting system.

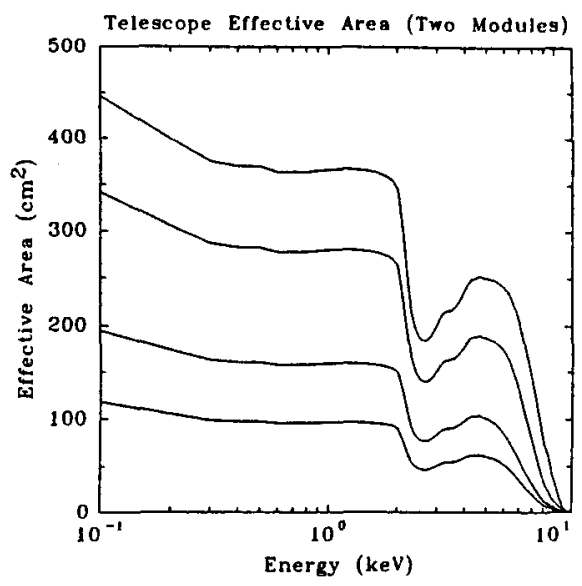

Figure 1: Total collecting area of two JET-X telescopes on-axis, and at off-axis angles of 7.5, 15.0 and 22.0 arcminutes.

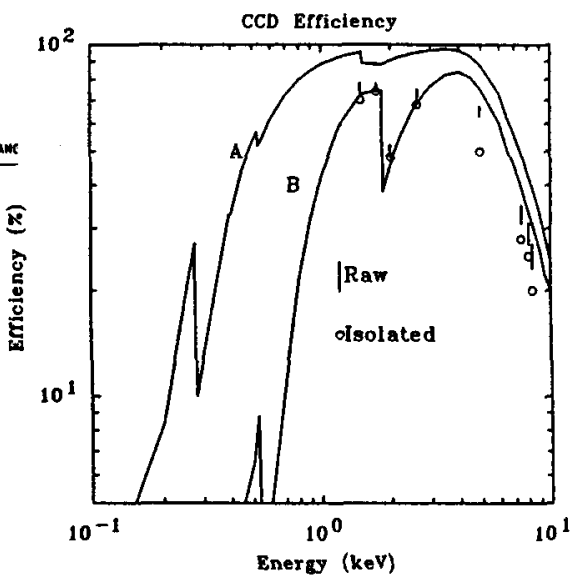

Figure 3: Current efficiency measurements for a $30 \mathrm{mi}-$ cron CCD. Both raw data (bars) and isolated pixel data with full spectral resolution (circles) are shown. For comparison curve $B$ shows the theoretical prediction for such a device. Curve $A$ shows the predicted efficiency for the JET-X baseline detector - a 40 micron thick, back-illuminated CCD including the attenuation of an aluminised light filter. 
between these two data sets. Figure 3 also shows the target response of the back-thinned device.

Figure 4 shows the energy resolution resulting from isolated event data with the pulse height distributions obtained by illuminating the CCD with characteristic $\mathrm{K}$ - emission lines of various metallic elements, with approximately 2000 photons incident in each line. This illustrates the effects of both energy resolution and efficiency. Neighbouring elements are well resolved down to an energy of $\sim 1 \mathrm{keV}$, whilst $\mathrm{H}-$ and $\mathrm{He}$-like ion lines are resolved down to an energy of $\sim 2 \mathrm{keV}$. Figure 5 shows that measured spectral resolutions agree precisely with the prediction from equation 1 for the measured CCD noise level of 10 electrons rms and the expected photoelectron Schott noise contribution for a Fano factor of 0.12 .

$$
\Delta \mathrm{E}(\mathrm{FWHM})=8.58\left(\mathrm{~N}^{2}+\mathrm{FE} / 3.65\right)^{0.5}
$$

where $\mathrm{N}=$ rms electron noise, $\mathrm{F}=$ Fano factor for silicon (0.12), and $\mathrm{E}=$ Photon Energy (keV).

Figure 6 shows the pulse height response (both before and after pixel anti-coincidence rejection is applied) to $\mathrm{Co}-60$ induced photo-electrons which are close to minimum ionising and therefore representative of the in-orbit background response of the CCD to protons and cosmic ray ions. A combination of anti-coincidence and energy veto allows an overall rejection efficiency of $>98 \%$.

Thinning and surface treatment processes are being developed to enhance the soft X-ray and short wavelength visible light responses. The CCD substrate can be etched back to the edge of the depletion region, so as to ensure high detection efficiency below $\sim 1 \mathrm{keV}$, with this surface treated to ensure that photo-electrons generated near the surface are pushed towards the depletion layer. A high concentration p-type layer is formed by ion implantation, and activated by annealing. The latest data show a dead surface layer of only $\sim 500$ Angstroms, which is confirmed by optical efficiency data at a wavelength of $400 \mathrm{~nm}$ where an efficiency of $60 \%$ is achieved. The absorption lengths of silicon at $0.5 \mathrm{keV}$ and $400 \mathrm{~nm}$ are comparable, indicating that excellent soft $\mathrm{X}$-ray efficiencies will be attainable. These techniques are now being applied to the deep depletion CCDs, and it is expected that the instrument detection efficiencies below $1 \mathrm{keV}$ will be determined largely by the aluminised lexan light block rather than the CCD itself. The CCD characteristics are summarised in Table 2

Table 2 CCD Detector Characteristics

$\begin{array}{lll}\begin{array}{l}\text { Energy Range } \\ \text { Geometric Area: } \\ \text { (single detector) }\end{array} & 0.2-10 \mathrm{keV} & \text { (>20\% efficiency) } \\ \quad\left(3^{*} 3 \text { CCD array) }\right. & 42 \times 30 \mathrm{~mm}^{2} \\ \text { Spatial Resolution } & <0.1 \mathrm{~mm} \\ \text { Background rejection } & >98.5 \% \\ \text { Spectral Resolution } & \text { See Figures } 4,5 \\ \text { Quantum Efficiency } & \text { See Figure 3 } \\ \text { Time Resolution: } & \\ \quad \text { (imaging mode) } & 20 \mathrm{~s} \\ \quad \text { (timing mode) } & 1 \mathrm{~ms} \\ \text { Operating Temperature } & 170-180 \mathrm{~K}\end{array}$




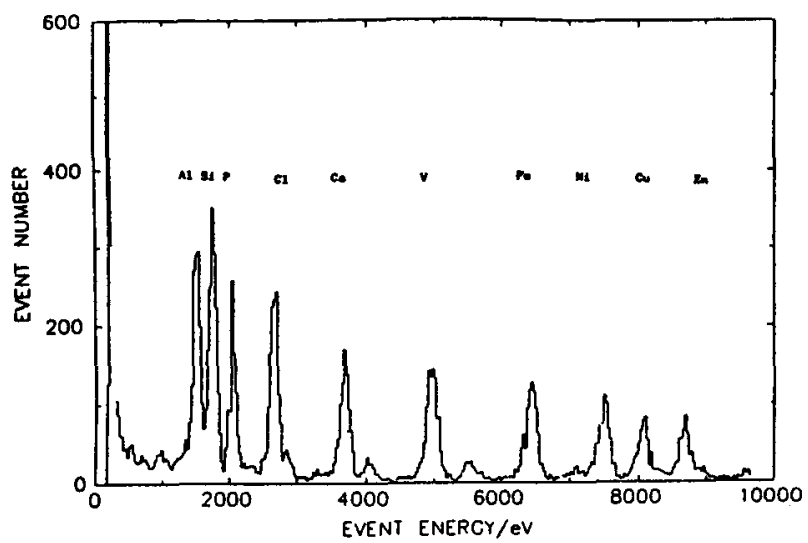

Figure 4: Measured pulse height response to a range of $\mathrm{K}$ emission lines of different elements.

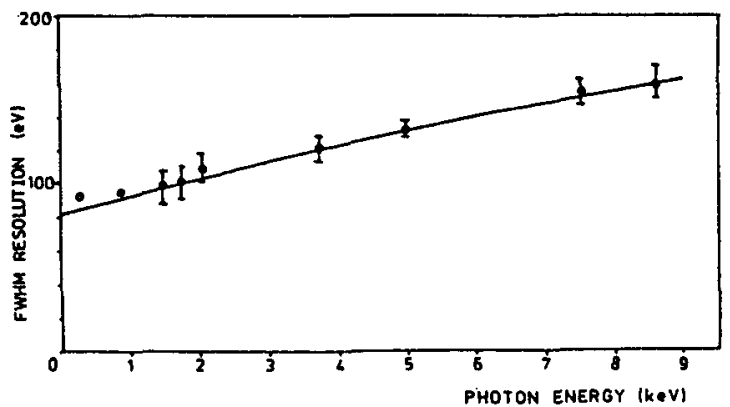

Figure 5: Comparison of measured and predicted energy resolution. Open circles represent predictions for the sub-keV performance in a back-illuminated CCD with same noise.

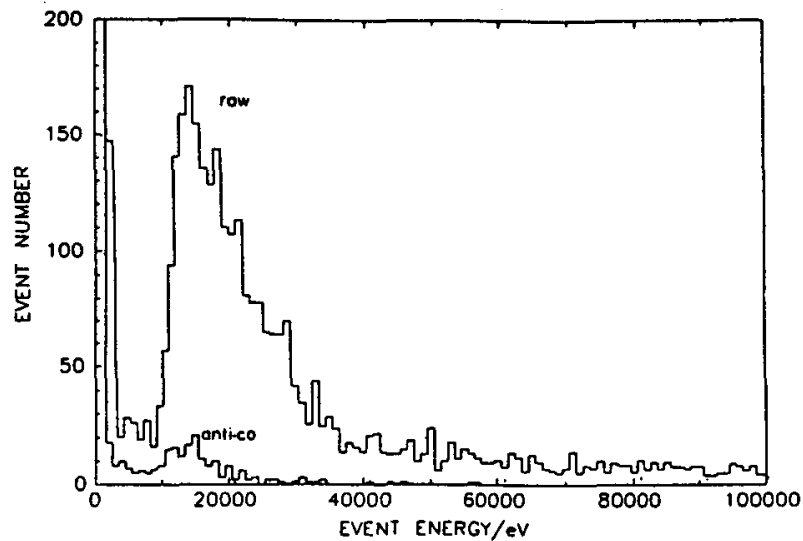

Figure 6: Pulse height response to charged particle background. Upper data - total energy deposition; lower data - residual data after pixel-pixel anti-coincidence. 


\subsection{Optical Monitor}

In addition to providing post facto attitude reconstruction, the optical monitor can simultaneously observe the optical counterparts of the X-ray sources and serendipitously study stellar microvariability. The first objective is directly related to the astrophysical understanding of $\mathrm{X}$-ray sources while the second allows JET-X to undertake a completely novel form of optical astronomy, because of the ability of JET-X to monitor continuously for up to $10^{5} \mathrm{~s}$.

The optical system consists of an F/5.7 Ritchey-Chretien telescope with a field corrector and an aperture of $30 \mathrm{~cm}$. The detector system is an optical CCD system which would have a high quantum efficiency leading to a photometric accuracy in a 1s integration of 0.04 mag for a star with $\mathrm{m}_{\mathrm{v}} \sim 15$. Read-out noise in the CCD would however lead to a limiting magnitude of $\mathrm{m}_{\mathrm{v}} \sim 20$, given the constraint on readout time imposed by the requirement that the monitor can also give attitude reconstruction measurements.

\subsection{Background Monitor}

The CCD detectors must be switched off for perigee passage and for occasions such as solar flares, when the background particle rates may be excessive. Whilst the former may be accommodated automatically within the mission timeline, the provision of a background monitor is required to allow the detection of a high background environment.

\subsection{Layered Synthetic Mono-structure}

A retractable synthetic multi-layer crystal may be included in one telescope module to give JET-X the additional capability of high resolution Bragg spectroscopy. The baseline design is a crystal constructed of $420 \mathrm{Ni} / \mathrm{C}$ layers, each $10 \AA$ thick. The crystal is shaped to intersect all $\mathrm{X}$-rays with a constant Bragg angle in the light-path from the mirrors. The Bragg angle is chosen such that the same focal plane detector as used for imaging studies can be used. An annular dispersed spectrum is then focussed on the CCD detector. The operating energy is $\sim 7 \mathrm{keV}$ with fine tuning provided by a small stepping motor. A resolution of $\frac{E}{\Delta E} \sim 350$ is currently possible, sufficient to resolve the individual components of the Fe XXV He-like triplet. It is believed that a resolution of 500 will be possible.

Preliminary measurements show that the throughput of this system is high, reflectivities of $\sim 30 \%$ having been attained. Further work will be undertaken to further improve this figure, the goal being a reflectivity of $50 \%$.

\section{SCIENTIFIC CAPABILITY}

\subsection{Instrument Performance}

The derived instrument performance assumes the telescope and detector data given in tables 1 and 2, a spatial resolution of 20 arcseconds, and a back-illuminated CCD. The anticipated background 
has three components :

a) The cosmic X-ray background: $N(E) d E=8 E^{-1.4} \mathrm{ph} \mathrm{cm}^{-2} \mathrm{~s}^{-1} \mathrm{keV}^{-1} \mathrm{sr}^{-1}$

b) A galactic diffuse X-ray background: $N(E) d E=120 E^{-1.0} \exp \left(\frac{-E}{0.3}\right) \mathrm{ph} \mathrm{cm}^{-2} \mathrm{~s}^{-1} \mathrm{keV}^{-1} \mathrm{sr}^{-1}$

c) Particle background for altitudes $>40000 \mathrm{~km}: 1.5 \times 10^{-2} \mathrm{cts}^{-1} \mathrm{~cm}^{-2}$ in the $0.1-10 \mathrm{keV}$ band after rejection.

\subsubsection{Point Source Sensitivity}

In Figure 7 the time taken to achieve a $5 \sigma$ detection of the source count rate integrated over the instrument passband is shown as a function of the $0.5-10 \mathrm{keV}$ flux for a source with a power-law spectrum of photon index 1.7, with low energy absorption corresponding to intervening cold matter with a neutral hydrogen column density of $10^{20} \mathrm{~cm}^{-2}$.

Also shown in the figure is the confusion limit (calculated using the 1 source per 30 beamwidth criterion) for a detector with 20 arcsec beamwidth. The limit has been extrapolated from the observed source number - flux relation measured for extra-galactic X-ray sources with the Einstein Observatory by assuming a continuing Euclidean population of sources. (The correction for the differing passbands of the instruments has been made assuming a power-law spectrum with photon index of 1.7 as is measured for the majority of local AGN).

The time taken to reach this confusion limit, roughly $10^{5} \mathrm{~s}$, is close to the expected orbital period of the SPECTRUM-X satellite, which therefore might be a typical exposure time, showing that the instrument throughput is well matched to its spatial resolution.

A by-product of the high sensitivity is that flux variability of approximately $10-20 \%$ on timescales of a few tens of seconds will be detectable in sources at flux levels of $\sim 1 \mu \mathrm{Jy}$. The simultaneous background measurement available with an imaging detector means that systematic errors will not substantially affect this type of measurement.

\subsubsection{Spectral Capability}

The energy resolution afforded by a CCD is well matched to the spectral properties of thermal plasmas with temperatures in the range $10^{6}-10^{8} \mathrm{~K}$. In addition to the strong iron lines at around 7 $\mathrm{keV}$, other strong lines from various ionisation stages of iron ( at $\sim 1 \mathrm{keV}$ ), silicon, sulphur, oxygen, calcium and argon fall within the passband of JET-X. JET-X is capable of resolving the resonance lines from the He- and $\mathrm{H}$ - like ionisation stages of these elements.

The time taken to measure lines at $7 \mathrm{kev}$ with equivalent widths of $2 \mathrm{keV}, 1 \mathrm{keV}$ and $100 \mathrm{eV}$, with respect to a power law continuum with a photon index of 1.7 , is shown in Figure 8 . A line with an equivalent width of $1 \mathrm{keV}$ can be detected at the 5 sigma level in $3 \times 10^{5} \mathrm{~s}$ for sources ten times brighter than the confusion limit (e.g. high redshift clusters). A value of $750 \mathrm{eV}$ is typical for cosmic plasmas with temperatures in the $2-8 \mathrm{keV}$ range. (At this observation length the line detection is still essentially photon limited and the limiting line intensity is $\sim 4 \times 10^{-6} \mathrm{ph} \mathrm{cm}^{-2} \mathrm{~s}^{-1}$ ). For sources down to $\sim 100 \mu \mathrm{Crab}$ strength lines of $100 \mathrm{eV}$ equivalent width can be detected. 
In addition to the medium resolution spectroscopy provided by the CCD detectors the inclusion of the layered synthetic monostructure (LSM) would enable studies at much higher resolution $\left(\frac{E}{\Delta E} \sim\right.$ $350-500$ ) in the region of the $\mathrm{Fe} \mathrm{K}$ line at $\sim 7 \mathrm{keV}$ to be made. For the current $30 \%$ reflective efficiency the limiting line sensitivity in a $10^{5} \mathrm{~s}$ observation would be $\sim 10^{-5} \mathrm{phcm}^{-2} \mathrm{~s}^{-1}$. For sources brighter than $10^{-12} \mathrm{erg} \mathrm{cm}^{-2} \mathrm{~s}^{-1}(1-10 \mathrm{keV}), 60$ times the EINSTEIN deep survey limit, lines with equivalent widths down to $1 \mathrm{keV}$ can be studied in detail.

\subsubsection{Spatial Resolution}

Objects larger than $500 \mathrm{Kpc}$, such as clusters of galaxies, will be resolved whatever their distance. Cooling flows, with typical extents of $100-200 \mathrm{Kpc}$ will also be spatially resolved at all distances, while individual galaxies would be resolved to redshifts of 0.5 . This is a significant advantage over, for example, a 2 arcminute resolution telescope.

\subsection{Astrophysical Impact}

The sensitivity and spectroscopic capability of JET-X are such that studies of astrophysical impor: tance for almost all classes of astronomical object can be made. Sufficiently large samples for each class of object will be observable that a major impact in all areas of astronomy, from planetary studies to cosmology, in addition to the studies of 'classical' X-ray sources will be possible. JET-X, however, has been designed primarily for the study of faint, distant X-ray sources of cosmological importance. Here a small sample of the key problems which can be addressed with JET-X are outlined.

\subsubsection{Faint Source Studies and the X-ray Background}

The high sensitivity of JET-X to point sources (for example, luminous AGNs will be detected to redshifts $\sim 4$ ) will enable source counts to be studied directly at flux levels $\sim 10$ times fainter than achieved with the EINSTEIN observatory, allowing a cross-comparison with the ROSAT X-ray telescope deep survey results in a complementary energy region.

If the $\log N-\log S$ is Euclidean to this flux level then JET-X will have imaged at least $50 \%$ of the diffuse X-ray background. On the other hand fluctuations analyses (Fabian 1981, Hamilton and Helfand 1987) of EINSTEIN data suggest that the source counts flatten at flux levels just below the EINSTEIN limits. This is at the flux level directly addressed by JET-X. Flattening of the source counts also implies that a new class of faint X-ray sources (e.g. young galaxies) may be required to explain the background. If these sources have a luminosity function similar to that suggested one should be visible in each $10^{5} \mathrm{~s}$ JET-X exposure.

JET-X can also measure the spectrum of the residual X-ray background over the $0.1-10 \mathrm{keV}$ band on scales down to less than 1 arc-minute. Comparison of this residual background with the spectra measured for individual populations of $\mathrm{X}$-ray emitters will further constrain possible models for the origin of the background. 


\subsubsection{Clusters}

The high spatial resolution, coupled with the continuum spectroscopic capability of JET-X, will enable detailed studies of the temperature and density profiles of the gas in clusters of galaxies to be determined. The gravitational potential to which the gas is responding may thus be mapped, allowing the determination of the distribution of both the visible and dark matter in clusters. Measurements of cluster gas parameters when coupled with measurements of the microwave decrement through the Sunyaev-Z'eldovitch effect will give model independent estimates of the cosmological parameters $H_{0}$ and $q_{0}$. Measurements of the angular diameters of cooling flows and clusters as a function of redshift will also lead to estimates of these parameters.

JET-X can test models of the evolution of clusters and the enrichment of the intra-cluster gas by measuring the abundance of heavy elements as a function of radius in clusters and as a function of redshift.

JET-X will strongly constrain the cooling-flow rates in nearby clusters, the distribution of flow rate with radius and will also determine the magnitude of flows in clusters at high redshift.

\subsubsection{Galaxies}

The study of X-ray emission from normal and star-burst galaxies has to-date been very limited. The EINSTEIN observatory results suggested that most elliptical galaxies contain large amounts of hot gas. JET-X will be able to detect the most luminous ellipticals out to redshifts of order 0.25 and obtain spectra for typical galaxies out to $100 \mathrm{Mpc}$ (e.g. the Coma cluster) in exposures of $<10^{5} \mathrm{~s}$. If, as is suggested, this gas is in hydrostatic equilibrium, measurements of the radial temperature and density distribution of the gas will allow the mass distribution of these galaxies to be determined.

Studies of cooling flows in nearby elliptical galaxies will also be possible. The 'canonical' wisdom that elliptical galaxies contained little or no gas and that consequently no star-formation was taking place is now untenable. X-ray observations of the cooling gas in these galaxies, particularly for a range of galaxy environments, will be essential in understanding the true picture.

The dominant emission from spiral galaxies, which in general have lower luminosities than ellipticals, is not from hot gas but from discrete sources, in particular X-ray binaries. Spectroscopy of these discrete sources will be possible throughout the local group. Comparative studies of the source populations will provide new data for evolutionary calculations through studies of their luminosity functions, distribution of orbital periods, location in the galaxies. The relationship of the X-ray source properties to their metallicities can also be investigated.

The spectral difference between the discrete sources and the diffuse ISM (if our galaxy is representative of spirals) will mean that studies of the ISM in galaxies at distances of up to $\sim 100 \mathrm{Mpc}$. will be possible. If these galaxies have gravitationally bound haloes with gas temperatures of $\sim 10^{6} \mathrm{~K}$, such as our galaxy, then it will be possible to derive the mass distribution within spirals as well as elliptical galaxies.

Star-burst galaxies have a much higher ratio of $L_{x}: L_{\text {opt }}$ than average and thus will be detectable out to distances of order $500 \mathrm{Mpc}$. The correlation between X-ray properties and the star-formation rate will mean that for an unbiased selection of star-burst galaxies the influences of effects such as inter-galaxy interaction can be studied. The relationship of $\mathrm{X}$-ray emission to the star-burst 
phenomenon in nearby galaxies will be important in undertanding the potential contribution of young galaxies to the $\mathrm{X}$-ray background.

\subsubsection{Active Galaxies}

The recent discovery (Nandra et al., 1989, Pounds et al., 1989) that AGN X-ray spectra are not simply featureless power-laws but that a substantial fraction show evidence for emission and absorption by cold iron imprinted on their spectra suggests another scientific area where the capabilities of JET-X are well-matched to the astrophysical problem. The good spectral resolution of the instrument below $1 \mathrm{keV}$ when coupled with the ability to precisely determine the intrinsic continuum will mean that detailed information about the physical condition and location of the absorbing material intrinsic to the AGN will be obtainable for a significant sample of active galaxies.

Some AGN such as MR2251-178 show evidence for absorption by partially ionised gas. As the intrinsic luminosity of the source changes so does the ionisation of this gas. The sensitivity of JET-X to the absorption edges, and hence ionisation states, of intermediate $\mathrm{Z}$ elements will be important in determining the location, density and abundance of this material.

A substantial fraction of AGN show excess emission at energies below $1 \mathrm{keV}$. These soft excesses are believed to be caused by emission from the accretion disc surrounding the central source. The low energy response of JET-X is sufficient to detect and investigate the temporal and spectroscopic behaviours of these soft excesses.

\section{Mission Profile}

In a lifetime of 5 years JET-X will be capable of making $\sim 10^{3}-10^{4}$ observations of durations between $10^{4}-10^{5} \mathrm{~s}$. In addition to the detailed information obtained on the few thousand prime targets JET$\mathrm{X}$ will serendipitously obtain information on $\sim 10^{5} \mathrm{X}$-ray sources. For at least $10 \%$ of these sources good quality spectra (sufficient, for example, to determine the power-law index of a serendipitous AGN to 0.1) will be obtained. Multi-colour broad-band photometry will be possible for most of the remainder. For comparison, previous imaging X-ray observatory instruments, provided little or no spectral information for most of the few thousand sources observed serendipitously and were not sensitive in the spectral band of most interest.

The ROSAT X-Ray Telescope has a sensitivity similar to JET-X but only at energies $<2 \mathrm{keV}$ and with limited spectral resolution. The ROSAT all-sky survey will provide invaluable information for optimising the scientific return from JET-X observations.

Scheduled for early 1993, well before either of the world class missions, AXAF and XMM, Spectrum- $X$ will be one of the first of several international missions to exploit the rich astrophysical potential of X-ray spectroscopy. In comparison with contemporary instruments, JET-X will have a lower throughput, but a much better limiting sensitivity, spectral and imaging resolution than X-SPECT (also included in the core payload of SPECTRUM-X). It will have comparable throughput but much better spatial resolution than the Japanese/US ASTRO-D and larger throughput and higher resolution than the imaging spectrometer on the Italian SAX missions likely to be in orbit on the same time scale. 


\section{References}

Chowanietz, E., Lumb, D.H. and Wells, A. 1986. Proc. SPIE.,597 381.

Citterio, O., Bonelli, G., Conti, G., Mattaini, E., Santambrogio, E., Sacco, B., Lanzara, E., Brauninger, H. \& Burkert, W. 1987. Proc. SPIE.830,139.

Fabian, A.C. 1981 . Ann. N.Y. Acad. Sci.375, 235.

Hamilton, T.T. \&z Helfand, D.J. 1987. Ap. J.318, 93.

Lumb, D.H. \& Holland A.D. 1989. Proc. SPIE.830,116.

Nandra, K., Pounds, K.A., Stewart, G.C., Fabian, A.C. \& Rees, M.J. 1989. MNRAS236,39P.

Pounds, K.A., Nandra, K., Stewart, G.C. \& Leighly, K. 1989. MNRAS Submitted.

Figure 7: Time required for a $5 \sigma$ detection of a point source for JET-X. Also shown are the confusion limits for 20 arc-seconds and 2 arc-minutes assuming a Euclidean extrapolation of the EINSTEIN $\log N-\log S$.

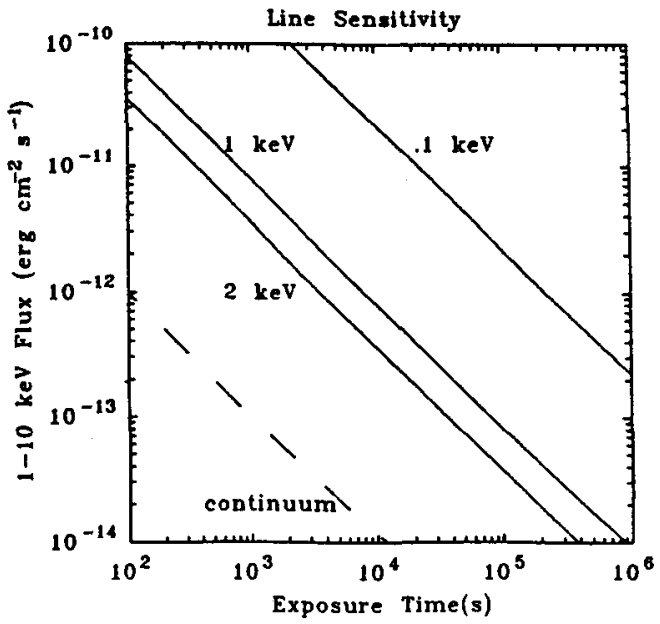

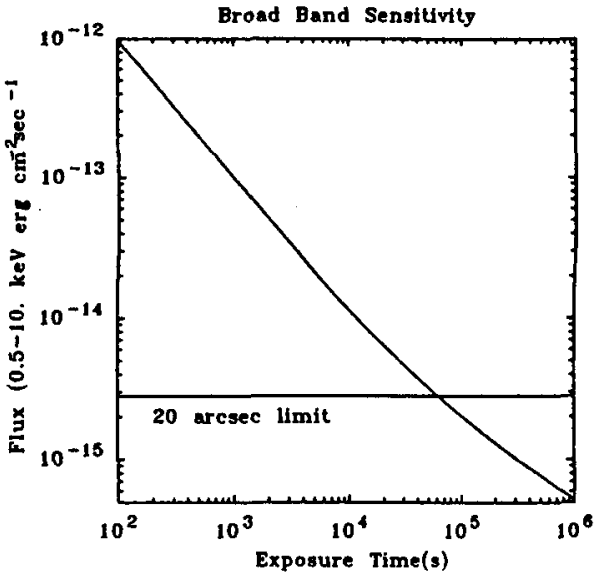

Figure 8: Time required by JET-X for a $5 \sigma$ detection of lines of varying equivalent widths as a function of the continuum intensity. 